\title{
Discrete and Continuous Linearizable Equations
}

\author{
S. LAFORTUNE ${ }^{\dagger}$ \\ LPTM et GMPIB, Université Paris VII \\ Tour 24-14, $5^{e}$ étage \\ 75251 Paris, France \\ B. Grammaticos \\ GMPIB, Université Paris VII \\ Tour 24-14, 5 étage \\ 75251 Paris, France \\ A. Ramani \\ CPT, Ecole Polytechnique \\ CNRS, UMR 7644 \\ 91128 Palaiseau, France
}

\begin{abstract}
We study the projective systems in both continuous and discrete settings. These systems are linearizable by construction and thus, obviously, integrable. We show that in the continuous case it is possible to eliminate all variables but one and reduce the system to a single differential equation. This equation is of the form of those singled-out by Painlevé in his quest for integrable forms. In the discrete case, we extend previous results of ours showing that, again by elimination of variables, the general projective system can be written as a mapping for a single variable. We show that this mapping is a member of the family of multilinear systems (which is not integrable in general). The continuous limit of multilinear mappings is also discussed.
\end{abstract}

† Permanent address: CRM, Université de Montréal, Montréal, H3C 3J7 Canada 


\section{INTRODUCTION}

The study of higher-order integrable systems is an interesting and open problem. It is all the more interesting when we realize that "higher" in this context means "higher then two". As a matter of fact the only instance where a complete classification of integrable systems can be given is in the case of second order differential equations. The study of equations of the form:

$$
w^{\prime \prime}=f\left(w^{\prime}, w, z\right)
$$

(where $f$ is polynomial in $w^{\prime}$, rational in $w$ and analytic in $z$ ) by Painlevé [1] and Gambier[2], based on the singularity structure of the solutions of (1.1), led to the complete classification of integrable equations of this type. Integrable in this setting means equations that can either

a) be integrated through quadratures

b) be reduced to a linear differential system through some local transformation

c) be integrated by isospectral methods involving linear integrodifferential equations. Calogero has coined the names C- and S-integrability for the two first types and third type of integrability respectively [3].

It is interesting to point out here that precisely the same three types of integrability were encountered when we undertook the the study of integrability of three-point non autonomous mappings of the form:

$$
\bar{x}=\frac{f_{1}(x)-f_{2}(x) \underline{x}}{f_{4}(x)-f_{3}(x) \underline{x}}
$$

where $f_{i}$ are polynomial in $x$ [4]. The main guide was the study of the singularity structure of the solutions of (1.2). However, contrary to the continuous case, the complete classifi-

cation of the integrable forms of (1.2) does not exist yet, although we are in possession of (at least) one discrete equation, for every member of the Painlevé/Gambier classification. The situation is further complicated in the discrete case by the fact that there exist two different kinds of discrete equations. As a matter of fact discrete equations can be of either additive or multiplicative type [5]. In the first case, the independent discrete variable appears linearly while in the second one the dependence is exponential.

In previous works $[6,7,8]$ we have tried to extend our results on integrable secondorder systems to equations of higher order. A large class of equations which are amenable to treatment is that corresponding to the second kind of integrability introduced above, namely integrability through linearisation. In particular we have concentrated on the discrete analogues of linearisable equations. In [9] we have studied the mapping trilinear in the $x$ 's:

$$
a_{1} \bar{x}_{1} x_{1} \underline{x_{1}}+a_{2} \bar{x}_{1} x_{1}+a_{3} \bar{x}_{1} \underline{x_{1}}+a_{4} \bar{x}_{1}+b_{1} x_{1} \underline{x_{1}}+b_{2} x_{1}+b_{3} \underline{x_{1}}+b_{4}=0
$$


and have shown that it contains as a subcase a mapping linearisable by reduction to a linear system. This case turned out to be the $N=2$ discrete projective Riccati equation that we introduced in [8], in the general $N$-dimensional case. Other approaches to higher-order systems can be found in [6].

In this paper we shall examine again the projective family of linearisable systems. We shall show in particular that it is possible to express the projective Riccati system as a single equation i.e. an equation for a single dependent variable, both in the continuous and in the discrete case. In the latter case the form of the system is a multilinear generalisation of (1.3). (A word of caution is due here. The terms "bi", "tri" or "multi"-linear should not be confused with the Hirota terminology. In the latter bilinear means a homogeneous quadratic expression while bilinear in our case means an expression where every variable enters linearly up to a highest degree of homogeneity two.) The multilinear mapping is, of course, much more general than the mere linearisable projective one. We illustrate this by studying some selected low-dimensional cases. Moreover we indicate how one can obtain the continuous limit of a multilinear mapping in a fairly general setting.

\section{Continuous Projective Systems}

We begin here by recalling what the continuous projective system is [10]. Our starting point will be the following first order linear ODE for the $(N+1)$-components vector $u$ :

$$
u^{\prime}=C u
$$

where $C$ is an $(N+1) \times(N+1)$ matrix depending on the independent variable $z$. We define the projective variables

$$
w_{i}=u_{i} / u_{N+1}, \quad i=1, \ldots, N \text {. }
$$

We then have

$$
w_{i}^{\prime}=\frac{u_{i}^{\prime}}{u_{N+1}}-\frac{u_{i} u_{N+1}^{\prime}}{u_{N+1}^{2}}
$$

which can be written in terms of the projective variables only. Thus the projective system reads:

$$
w_{i}^{\prime}=\left(\sum_{j=1}^{N} C_{i j} w_{j}+C_{i N+1}\right)-w_{i}\left(\sum_{j=1}^{N} C_{N+1 j} w_{j}+C_{N+1 N+1}\right), \quad 1 \leq i \leq N .
$$

For the $N=1$ case, we get the Riccati equation

$$
w^{\prime}=a w^{2}+b w+c
$$


where $a, b$ and $c$ are functions of the independent variable $z$. This equation is special as far as singularity analysis is concerned. If we consider a first order equation of the form

$$
w^{\prime}=f(w, z)
$$

where $P$ is a polynomial in $w$ and analytic in $z$, the only nonlinear equation of the form (2.5) satisfying the Painlevé property is the Riccati.

In the case of the $N=2$ projective system, we can eliminate $w_{2}$ in the system $(2.3)$. The equation then reads:

$$
\left(b_{1} w_{1}^{\prime}+b_{2}\right) w_{1}^{\prime \prime}+b_{3} w_{1}^{\prime 2}+\left(b_{4} w_{1}+b_{5}\right) w_{1}^{\prime}+b_{6} w_{1}^{3}+b_{7} w_{1}^{2}+b_{8} w_{1}+b_{9}=0
$$

where the $b_{k}$ 's are functions of the $C_{i j}$ 's. This is an equation which corresponds to one of the fifty equations of the Painlevé-Gambier classification. After a suitable change of variable, we write it in its canonical form [11]:

$$
w^{\prime \prime}+3 w w^{\prime}+w^{3}+\phi(z)\left(w^{\prime}+w^{2}\right)=0 .
$$

where $\phi$ is a free function of the independent variable $z$. We will now show that, in general, the continuous projective system can be written as one single equation. From (2.3), we rewrite the first-order equation for $w_{1}$ :

$$
w_{1}^{\prime}=\sum_{j=1}^{N} C_{1 j} w_{j}+C_{1 N+1}-w_{1}\left(\sum_{j=1}^{N} C_{N+1 j} w_{j}+C_{N+1 N+1}\right) .
$$

Then, differentiating both sides of (2.8), and keeping track of the $w_{j}$ 's, $j \neq 1$

$$
\begin{aligned}
& w_{1}^{\prime \prime}=\sum_{j=2}^{N} C_{1 j}^{\prime} w_{j}+\sum_{j=2}^{N} C_{1 j} w_{j}^{\prime}-w_{1}^{\prime}\left(\sum_{j=1}^{N} C_{N+1 j} w_{j}\right) \\
& -w_{1}\left(\sum_{j=2}^{N} C_{N+1 j} w_{j}^{\prime}+\sum_{j=2}^{N} C_{N+1 j}^{\prime} w_{j}\right)+D\left(w_{1}^{\prime}, w_{1}, z\right)
\end{aligned}
$$

where $D$ depends only on $w_{1}^{\prime}, w_{1}$ and the independent variable $z$. We see here that the only terms with degree higher than linear in the $w_{k}$ 's, $k \neq 1$, are the $w_{j}^{\prime}$ terms. But these appear only through the combination

$$
\sum_{j=2}^{N}\left(C_{1 j}-w_{1} C_{N+1 j}\right) w_{j}^{\prime}
$$


Using (2.3) and keeping only the potentially nonlinear terms coming from the second term in the lhs, we see that we should only worry about an expression of the form: $\left(\sum_{j=2}^{N}\left(C_{1 j}-w_{1} C_{N+1 j}\right) w_{j}\right)\left(\sum_{k=1}^{N} C_{N+1 k} w_{k}+C_{N+1 N+1}\right)$ which is, potentially, nonlinear in the $w_{k}$ 's, $k \neq 1$. Note however, that the first factor can be expressed in terms of $w_{1}^{\prime}$ and $w_{1}$ only, since this is precisely the combination of $w_{j}$ 's that enter in (2.8). Thus by replacing this factor by its expression in terms of $w_{1}^{\prime}$ and $w_{1}$ the only source of $w_{k}$ 's, $k \neq 1$, comes from the second factor and thus no nonlinearities are introduced.

Regrouping all terms we find:

$$
w_{1}^{\prime \prime}=\sum_{j=1}^{N} f_{2 j}\left(w_{1}, w_{1}^{\prime}, z\right) w_{j}+f_{2 N+1}\left(w_{1}, w_{1}^{\prime}, z\right)
$$

where the $f_{2 j}$ 's are some definite functions of $w_{1}, w_{1}^{\prime}$ and $z$. In the general case, it is not difficult to see that

$$
w_{1}^{(n)}=\sum_{j=1}^{N} f_{n j}\left(w_{1}, w_{1}^{\prime}, \ldots, w_{1}^{(n-1)}, z\right) w_{j}+f_{n N+1}\left(w_{1}, w_{1}^{\prime}, \ldots, w_{1}^{(n-1)}, z\right), \quad n \in \mathbb{Z}
$$

So, we have a linear and inhomogeneous equation for the vector $W$ with components $w_{1}$, $w_{2}, \ldots, w_{N}$ :

$$
D W+E=0
$$

where $D$ is an $N \times N$ matrix and $E$ is an $N$ components vector defined by

$$
\begin{gathered}
D_{i j}=f_{i j} \\
E_{i}=f_{i N+1}-w_{1}^{(i)} .
\end{gathered}
$$

Hence, the $N^{\text {th }}$ order equation satisfied by $w_{1}$ reads

$$
\sum_{j=1}^{N} \operatorname{adj}(\mathrm{D})_{1 j}\left(w_{1}^{(j)}-f_{j N+1}\right)-\operatorname{det}(D) w_{1}=0
$$

Thus the general projective system can indeed be written as an equation for a single variable. For instance, in the case $N=3$, we get the following third order equation [12]

$$
w^{\prime \prime \prime}=\frac{3 / 2 w^{\prime \prime 2}+w^{\prime \prime}\left(g_{1}(z) w w^{\prime}+g_{2}(z) w^{\prime}+P_{1}(w)\right)+P_{3}\left(w, w^{\prime}\right)+g_{3}(z) w^{4}}{w^{\prime}+P_{2}(w)}
$$

where $P_{1}(w)$ and $P_{2}(w)$ are polynomials of degree two in $w, P_{3}\left(w, w^{\prime}\right)$ is a polynomial of degree three in $w$ and $w^{\prime}$ and the $g_{i}$ 's are functions of the independent variable $z$. The polynomials have coefficients depending on $z$. 
For higher $N$ 's, we can in principle obtain the corresponding $N$-th order equation for a single variable. However, the computations soon become unmanageable. Since we cannot give a detailed general expression, we must content ourselves with the global structure of this equation. For order $N$, we have:

$$
w^{(N)}=M\left(w^{(N-1)}\right)^{2}+P w^{(N-1)}+Q
$$

where $M, P$ and $Q$ are rational functions of $w^{(N-2)}, w^{(N-3)}, \ldots, w$, and analytic in $z$. In particular, for $M$ we have:

$$
M=\frac{1-1 / n}{w^{(N-2)}+R}
$$

where $n=1-N$ and $R$ depends only on $w^{(N-3)}, \ldots, w, z$.

What is interesting is that equation (2.17) together with (2.18) is exactly one of the forms given by Painlevé [13] for $N$-th order equations having the Painlevé property. According to Painlevé, when $M$ has just the simple expression (2.18) then $P$ and $Q$ must be, as functions of $w^{(N-2)}$, ratios of a polynomial of degree at most one for $P$ and at most three for $Q$, divided by the same denominator as that of $M$. We expect this property to hold for our projective systems, since they are integrable and thus should have the Painlevé property. We have indeed checked that this holds true at least up to order 5 .

\section{Discrete Projective Systems}

In the discrete case, the projective system comes from the following two-points linear system for the $(N+1)$-component vector $u$ :

$$
\bar{u}=M u
$$

where $M$ is an $(N+1) \times(N+1)$ matrix with elements depending on the discrete independent variable $n$ and where we use the notation $u=u(n)$ and $\bar{u}=u(n+1)$. We define the projective variables:

$$
w_{i}=u_{i} / u_{N+1}, i=1, \ldots, N .
$$

We then get the projective system [8]:

$$
\bar{w}_{i}=\frac{\sum_{j=1}^{N} M_{i j} w_{j}+M_{i N+1}}{\sum_{j=1}^{N} M_{N+1 j} w_{j}+M_{N+1 N+1}} .
$$

In the case $N=1$, we get the single equation

$$
\bar{w}_{1}=\frac{M_{11} w_{1}+M_{12}}{M_{21} w_{1}+M_{22}}
$$


which is the discrete form of the Riccati equation. Mapping (3.4) is very special as far as integrability is concerned [9]. Consider mappings of the form

$$
\bar{v}=f(v, n)
$$

where $f$ is some rational function in $v$. We can easily convince ourselves that the only non-polynomial mapping of the form (3.5) that has confined singularities is a mapping of the form

$$
\bar{v}=A_{0}+\sum_{k} \frac{A_{k}}{\left(a_{k} v+d_{k}\right)^{m_{k}}},
$$

with $m_{k}$ integer. However if we consider the "backward" evolution towards diminishing $n$ then (3.6), $v$ expressed in terms of $\bar{v}$, is not rational. The only nonlinear mapping with confined singularities that is rational in both directions is just

$$
\bar{v}=A_{0}+\frac{A_{1}}{a_{1} v+d_{1}} \equiv \frac{a v+b}{c v+d} .
$$

In the $N=2$ case, we can write the projective system as a single three-point mapping for $w_{1}$ [9]. To do this, we write the equation (3.1) for $\bar{u}$ and also the equation satisfied by $\underline{u}:$

$$
\begin{gathered}
\bar{u}=M u \\
\underline{u}=P u
\end{gathered}
$$

where $M$ and $P$ are two $n$-dependent $3 \times 3$ matrices and $P$ is given in terms of $M$ by

$$
P=\underline{M}^{-1}
$$

¿From (3.8a) and (3.8b) we have the two equations

$$
\begin{gathered}
\bar{w}_{1}=\frac{M_{11} w_{1}+M_{12} w_{2}+M_{13}}{M_{31} w_{1}+M_{32} w_{2}+M_{33}} \\
\underline{w_{1}}=\frac{P_{11} w_{1}+P_{12} w_{2}+P_{13}}{P_{31} w_{1}+P_{32} w_{2}+P_{33}} .
\end{gathered}
$$

¿From the system (3.10) we easily write the three-point mapping satisfied by $w_{1}$ :

$$
a_{1} \bar{w}_{1} w_{1} \underline{w_{1}}+a_{2} \bar{w}_{1} w_{1}+a_{3} \bar{w}_{1} \underline{w_{1}}+a_{4} \bar{w}_{1}+b_{1} w_{1} \underline{w_{1}}+b_{2} w_{1}+b_{3} \underline{w_{1}}+b_{4}=0
$$

where the $a_{i}$ 's and the $b_{i}$ 's are functions of the components of $M$ and $P$. We note that (3.11) is of the form of the most general multilinear equation in $\bar{w}_{1}, w_{1}$ and $w_{1}$ one can write. 
In the general case, we will prove here that one can still write the projective system as one single equation. We will also see that the mapping we obtain has also the form of the most general multilinear equation one can write in terms of $w_{1}$ and its $N$ first upshifts.

For arbitrary $N$ we first write a system of the form (3.1) for $\bar{u}$ and the systems satisfied by the $N$ first upshifts of $u$ :

$$
\begin{gathered}
\bar{u}=M_{1} u \\
\overline{\bar{u}}=M_{2} u \\
\vdots \\
\overline{\overline{\bar{u}}}=M_{N} u
\end{gathered}
$$

where the last object is the $N$-th upshift of $u$ and the $M_{i}$ 's are $(N+1) \times(N+1) n$ dependent matrices. For $i>2, M_{i}$ can be recursively expressed in terms of $M_{1}$ and its upshifts through $M_{i}=\bar{M}_{i-1} M_{1}$. We then get the following mappings for $w_{1}=u_{1} / u_{N+1}$ :

$$
\begin{gathered}
\bar{w}_{1}=\frac{\sum_{j=1}^{N}\left(M_{1}\right)_{1 j} w_{j}+\left(M_{1}\right)_{1 N+1}}{\sum_{j=1}^{N}\left(M_{1}\right)_{N+1 j} w_{j}+\left(M_{1}\right)_{N+1 N+1}} \\
\overline{\bar{w}}_{1}=\frac{\sum_{j=1}^{N}\left(M_{2}\right)_{1 j} w_{j}+\left(M_{2}\right)_{1 N+1}}{\sum_{j=1}^{N}\left(M_{2}\right)_{N+1 j} w_{j}+\left(M_{2}\right)_{N+1 N+1}} \\
\vdots \\
\overline{\bar{w}}_{1}=\frac{\sum_{j=1}^{N}\left(M_{N}\right)_{1 j} w_{j}+\left(M_{N}\right)_{1 N+1}}{\sum_{j=1}^{N}\left(M_{N}\right)_{N+1 j} w_{j}+\left(M_{N}\right)_{N+1 N+1}} .
\end{gathered}
$$

To write this system in a compact way, we define:

$$
\begin{gathered}
v_{0} \equiv w_{1} \\
v_{1} \equiv \bar{w}_{1} \\
v_{2} \equiv \overline{\bar{w}}_{1} \\
\vdots \\
v_{N} \equiv \overline{\bar{w}}_{1}
\end{gathered}
$$

and system (3.13) can be rewritten as

$$
v_{i}=\frac{\sum_{j=1}^{N}\left(M_{i}\right)_{1 j} w_{j}+\left(M_{i}\right)_{1 N+1}}{\sum_{j=1}^{N}\left(M_{i}\right)_{N+1 j} w_{j}+\left(M_{i}\right)_{N+1 N+1}} i=1, \ldots, N
$$


The system (3.15) is an inhomogeneous linear equation for the vector $W$ with components $w_{j}, j=1, \ldots, N$ :

$$
C W+B=0
$$

where $C$ is an $N \times N$ matrix and $B$ is an $N$-component vector defined by

$$
\begin{gathered}
C_{i j}=\left(M_{i}\right)_{1 j}-v_{i}\left(M_{i}\right)_{N+1 j} \\
B_{i}=\left(M_{i}\right)_{1 N+1}-v_{i}\left(M_{i}\right)_{N+1 N+1} \\
1 \leq i, j \leq N .
\end{gathered}
$$

Solving (3.16) for $w_{1} \equiv v_{0}$, we obtain:

$$
\operatorname{det}(C) v_{0}+\sum_{j=1}^{N}(\operatorname{adj}(\mathrm{C}))_{1 j} B_{j}=0
$$

¿From the definitions (3.17), we see that $\operatorname{det}(C)$ will generically be a general multilinear expression in the $v_{i}$ 's (which are just the different shifts of $w_{1}$ ). The first term of equation (3.18) will include all the multilinear terms containing $v_{0} \equiv w_{1}$. Moreover $\operatorname{adj}(\mathrm{C})_{1 j}$ is a general multilinear expression in $v_{i}$ for $i=1,2, \ldots, j-1, j+1, \ldots, N$. The second term of equation (3.18) will thus be in the form of a general multilinear expression in the shifts of $w_{1}$, the unshifted $w_{1}$ excepted. Hence, we can see that (3.18) has indeed the form of the most general multilinear equation in $w_{1}$ and its $N$ first upshifts, except that the coefficients are not all free.

\section{Confinement of the projective Mapping}

In this section we are considering the same projective system as in Section 3. For the cases $N=2,3$ it is already known that mapping (3.18) confines in one step [9]. The case $N=2$ simply corresponds to the Riccati mapping (3.4). We will prove here that confinement [14] occurs in one step for arbitrary value of $N$.

First, we prove the following formula

$$
\frac{\partial\left(\bar{w}_{1}, \bar{w}_{2}, \ldots, \bar{w}_{N}\right)}{\partial\left(w_{1}, w_{2}, \ldots, w_{N}\right)}=\operatorname{det}\left(M_{1}\right)\left(\frac{u_{N+1}}{\bar{u}_{N+1}}\right)^{N+1}
$$

To prove this we first have to use the chain rule:

$$
\begin{aligned}
& \frac{\partial\left(\bar{u}_{1}, \bar{u}_{2}, \ldots, \bar{u}_{N+1}\right)}{\partial\left(u_{1}, u_{2}, \ldots, u_{N+1}\right)}= \\
& \frac{\partial\left(\bar{u}_{1}, \bar{u}_{2}, \ldots, \bar{u}_{N+1}\right)}{\partial\left(\bar{w}_{1}, \bar{w}_{2}, \ldots, \bar{w}_{N}, \bar{u}_{N+1}\right)} \frac{\partial\left(\bar{w}_{1}, \bar{w}_{2}, \ldots, \bar{w}_{N}, \bar{u}_{N+1}\right)}{\partial\left(w_{1}, w_{2}, \ldots, w_{N}, u_{N+1}\right)} \frac{\partial\left(w_{1}, w_{2}, \ldots, w_{N}, u_{N+1}\right)}{\partial\left(u_{1}, u_{2}, \ldots, u_{N+1}\right)}
\end{aligned}
$$


Then using (3.2) and (3.3), we easily calculate that

$$
\begin{gathered}
\frac{\partial\left(\bar{u}_{1}, \bar{u}_{2}, \ldots, \bar{u}_{N+1}\right)}{\partial\left(\bar{w}_{1}, \bar{w}_{2}, \ldots, \bar{w}_{N}, \bar{u}_{N+1}\right)}=\bar{u}_{N+1}^{N} \\
\frac{\partial\left(\bar{w}_{1}, \bar{w}_{2}, \ldots, \bar{w}_{N}, \bar{u}_{N+1}\right)}{\partial\left(w_{1}, w_{2}, \ldots, w_{N}, u_{N+1}\right)}=\frac{\partial\left(\bar{w}_{1}, \bar{w}_{2}, \ldots, \bar{w}_{N}\right)}{\partial\left(w_{1}, w_{2}, \ldots, w_{N}\right)} \frac{\bar{u}_{N+1}}{u_{N+1}} \\
\frac{\partial\left(w_{1}, w_{2}, \ldots, w_{N}, u_{N+1}\right)}{\partial\left(u_{1}, u_{2}, \ldots, u_{N}, u_{N+1}\right)}=u_{N+1}^{-N}
\end{gathered}
$$

Equation (4.1) is then proven. But $M_{1}$ can never be singular. Indeed, if it were singular for some $n$, then the mapping would always lose a degree of freedom at $n$, independently of the initial conditions. This would be a 'fixed' singularity, which we do not consider. Hence, in what follows, we will always consider that the Jacobian between the $\bar{w}_{i}$ 's and the $w_{i}$ 's is nonzero: the mapping giving the $\bar{w}_{i}$ 's from the $w_{i}$ 's is never singular.

What we mean by a singularity in the $v$-mapping is that the information in the variables of the mapping is not the full one, i.e. there is a loss of information at step $N$.

So saying that the singularity enters with the set $\left(v_{1}, v_{2}, \ldots, v_{N}\right)$, (which is the first one where $v_{N}$ appears) means

$$
\frac{\partial\left(v_{1}, v_{2}, \ldots, v_{N}\right)}{\partial\left(v_{0}\left(\equiv w_{1}\right), v_{1}, v_{2}, \ldots, v_{N-1}\right)}=(-1)^{N} \frac{\partial v_{N}}{\partial v_{0}}=0
$$

Using the chain rule as well as (4.1) this writes:

$$
\frac{\partial\left(v_{1}, v_{2}, \ldots, v_{N}\right)}{\partial\left(\bar{w}_{1}, \bar{w}_{2}, \ldots, \bar{w}_{N}\right)} \operatorname{det} M_{1}\left(\frac{u_{N+1}}{\bar{u}_{N+1}}\right)^{N+1}\left(\frac{\partial\left(v_{0}, v_{1}, v_{2}, \ldots, v_{N-1}\right)}{\partial\left(w_{1}, w_{2}, \ldots, w_{N}\right)}\right)^{-1}=0
$$

Thus a singularity at step $N$ implies that:

$$
\frac{\partial\left(v_{1}, v_{2}, \ldots, v_{N}\right)}{\partial\left(\bar{w}_{1}, \bar{w}_{2}, \ldots, \bar{w}_{N}\right)}=0
$$

(note that $v_{1}=\bar{w}_{1}$ ). We assume that the singularity actually occurs there for the first time i.e. that:

$$
\frac{\partial\left(v_{0}, v_{1}, v_{2}, \ldots, v_{N-1}\right)}{\partial\left(w_{1}, w_{2}, \ldots, w_{N}\right)} \neq 0
$$

Thus the appearence of the singularity is related to the fact that the $N$ quantities $\left(v_{1}, v_{2}, \ldots, v_{N}\right)$ do not carry enough information on the $\left(\bar{w}_{1}, \bar{w}_{2}, \ldots, \bar{w}_{N}\right)$. Though the $w_{i}$ 's have all the information at all steps, some is lost in the particular combination $\left(v_{1}, v_{2}, \ldots, v_{N}\right)$. At step $N+1$ the relevant Jacobian does not vanish.

$$
\frac{\partial\left(v_{2}, \ldots, v_{N}, v_{N+1}\right)}{\partial\left(\overline{\bar{w}}_{1}, \overline{\bar{w}}_{2}, \ldots, \overline{\bar{w}}_{N}\right)} \neq 0
$$


Indeed, this Jacobian contains information about $M_{N+1}$ and thus the $N$-th upshift of $M_{1}$, which is independent of all others, through, and only through $v_{N+1}$. Thus since this quantity is independent from the others, the Jacobian cannot vanish. Formally, one should write the upshift of (3.18) as a multilinear equation of order $N+1$ in the $v_{1}, v_{2}, \ldots, v_{N+1}$ and solve for $v_{N+1}$ as:

$$
v_{N+1}=\frac{F\left(v_{1}, v_{2}, \ldots, v_{N}\right)}{G\left(v_{1}, v_{2}, \ldots, v_{N}\right)}
$$

where $F$ and $G$ are multilinear functions of their arguments. But if $v_{N+1}$ were actually a function of $\left(v_{1}, v_{2}, \ldots, v_{N}\right)$, then it would follow that the $\left(v_{1}, v_{2}, \ldots, v_{N+1}\right)$ would not contain more information than $\left(v_{1}, v_{2}, \ldots, v_{N}\right)$ and thus $\left(v_{2}, \ldots, v_{N+1}\right)$ certainly not more either, in contradiction with (4.10). It follows that if (4.8) holds, (4.11) must be of the form $0 / 0$ (i.e. the upshift of (3.18) is identically zero as a function of $v_{N+1}$ ). In fact, (4.10) implies that the lost degree of freedom has been recovered and that the singularity is confined already at this step.

In the case $N=2$, the downshift of (4.8) reduces to

$$
v_{0}\left(\left(M_{1}\right)_{11}\left(M_{1}\right)_{32}-\left(M_{1}\right)_{12}\left(M_{1}\right)_{31}\right)+\left(M_{1}\right)_{13}\left(M_{1}\right)_{32}-\left(M_{1}\right)_{12}\left(M_{1}\right)_{33}=0
$$

This implies that

$$
v_{1}=\bar{w}_{1}=\frac{\left(M_{1}\right)_{12}}{\left(M_{1}\right)_{32}}
$$

and thus does not depend on $\underline{w}_{1}$. In the case $N=2,(3.15)$ is only a system of two equations. The second equation of (3.15) gives $v_{2}$ in terms of $v_{1}$ and $v_{0}$ :

$$
\begin{gathered}
v_{2}\left(v_{1} v_{0}\left(\left(M_{1}\right)_{32}\left(M_{2}\right)_{31}-\left(M_{1}\right)_{31}\left(M_{2}\right)_{32}\right)+v_{1}\left(\left(M_{1}\right)_{32}\left(M_{2}\right)_{33}-\left(M_{1}\right)_{33}\left(M_{2}\right)_{32}\right)\right. \\
\left.\quad+v_{0}\left(\left(M_{1}\right)_{11}\left(M_{2}\right)_{32}-\left(M_{1}\right)_{12}\left(M_{2}\right)_{31}\right)+\left(M_{1}\right)_{13}\left(M_{2}\right)_{32}-\left(M_{1}\right)_{12}\left(M_{2}\right)_{33}\right) \\
+v_{1} v_{0}\left(\left(M_{1}\right)_{31}\left(M_{2}\right)_{12}-\left(M_{1}\right)_{32}\left(M_{2}\right)_{11}\right)+v_{1}\left(\left(M_{1}\right)_{33}\left(M_{2}\right)_{12}-\left(M_{1}\right)_{32}\left(M_{2}\right)_{13}\right) \\
+v_{0}\left(\left(M_{1}\right)_{12}\left(M_{2}\right)_{11}-\left(M_{1}\right)_{11}\left(M_{2}\right)_{12}\right)+\left(M_{1}\right)_{12}\left(M_{2}\right)_{13}-\left(M_{1}\right)_{13}\left(M_{2}\right)_{12}=0
\end{gathered}
$$

Implementing (4.12) and (4.13) into (4.14), we find that the coefficient of $v_{2}$ and the rest of the equation are both zero. So, formally, $v_{2}$ is indeed $0 / 0$ and a detailed calculation shows that it does depend on $\underline{w}_{1}$.

\section{Continuous limits of multilinear mappings}

In the previous sections, we have seen that the projective systems can always be written as multilinear mappings of a single variable. Since the continuous system can also be written as a single differential equation, it is interesting to investigate the continuous 
limit of the multilinear mappings. We shall do this in a general setting i.e. without imposing to the mapping the integrability constraints associated to its projective character.

Our main assumption is that the discrete variable $u$ coincides with the continuous variable $w$ at the continuous limit. This will indeed turn out to be the right choice. We start by considering that we have a mapping of order $N$ (i.e. a $(N+1)$-point mapping). At the continuous limit, this would lead to an $N$-th order differential equation. Moreover, the highest nonlinearity is of degree $(N+1)$. Given its structure, the multilinear mapping is invariant under the transformation $u \rightarrow 1 / u$. It turns out that the 'richest' terms in the mapping are the 'middle' ones: they have the largest number of terms of the same homogeneity. More precisely, if the order $N$ of the mapping is odd, the degree of the middle term is $(N+1) / 2$. If the order is even then there are two 'middle' terms with degree $N / 2$ and $N / 2+1$. It suffices in this case to consider one of them, say the one of degree $N / 2$. Indeed it turns out that the contribution of the other 'middle' term can be absorbed into that of the first term through a translation and subsequent inversion of the continuous variable. Since these 'middle' terms have the highest nonlinearity combined with the highest degree they are the ones that play the dominant role in the differential equation obtained.

Let us make these considerations more precise by analyzing specific examples.

We start with the simplest possible case of order $N=1$, the discrete Riccati equation. We have:

$$
\alpha \bar{u} u+\beta \bar{u}+\gamma u+\delta=0
$$

Clearly the two 'middle' terms $\{\bar{u}, u\}$ can be combined to produce a first derivative $w^{\prime}$ and the nonlinearity comes from the first term $\bar{u} u \rightarrow w^{2}$. Let us now consider the somewhat more interesting cubic case corresponding to $N=2$

$$
\alpha \bar{u} u \underline{u}+\beta \bar{u} u+\gamma \bar{u} \underline{u}+\delta \underline{u}+\zeta \bar{u}+\theta u+\kappa \underline{u}+\lambda=0
$$

The continuous limit is obtained through $u \rightarrow w, \bar{u}=w+\varepsilon w^{\prime}+\varepsilon^{2} w^{\prime \prime} / 2, \underline{u}=w-\varepsilon w^{\prime}+$ $\varepsilon^{2} w^{\prime \prime} / 2$. We assume that at leading order the coefficients behave like: $\beta=-B / \varepsilon^{2}+\mathcal{O}(1 / \varepsilon)$, $\gamma=2 B / \varepsilon^{2}+\mathcal{O}(1 / \varepsilon), \delta=-B / \varepsilon^{2}+\mathcal{O}(1 / \varepsilon), \zeta=A / \varepsilon^{2}+\mathcal{O}(1 / \varepsilon), \theta=-2 A / \varepsilon^{2}+\mathcal{O}(1 / \varepsilon)$, $\kappa=A / \varepsilon^{2}+\mathcal{O}(1 / \varepsilon)$. We find then that the second-degree terms lead to $B\left(w w^{\prime \prime}-2 w^{\prime 2}\right)$ at lowest order in $\varepsilon$, while the first-degree one give simply $A w^{\prime \prime}$. Translating $w$ by $A / B$ we can absorb the $A w^{\prime \prime}$ term into $B\left(w w^{\prime \prime}-2 w^{\prime 2}\right)$. We define now $x=(w+A / B)^{-1}$ and obtain as only dominant term $B x^{\prime \prime}$. The detailed continuous limit of (5.2) must take into account the precise form of the $\alpha, \beta, \ldots$ coefficients. It was performed in [9] where we have shown that the continuous limit is

$$
x^{\prime \prime}+3 x x^{\prime}+x^{3}+q(z)\left(x^{\prime}+x^{2}\right)+r(z) x+s(z)=0 .
$$


This is precisely the form of the linearisable differential equation obtained by Painlevé at order two.

In the case of the $N=3$ multilinear mapping there exists only one contribution at dominant order. Its continuous limit, obtained along lines similar to those of the $N=2$ case, leads to a dominant term $w^{\prime \prime \prime} w^{\prime}-\frac{3}{2} w^{\prime \prime 2}$. Since this is the only dominant contribution it is interesting to study the subdominant ones. We find three groups of terms: $a w^{\prime \prime \prime}\left(w^{2}-\right.$ $\left.6 w w^{\prime} w^{\prime \prime}+6 w^{\prime 3}\right)+b w^{\prime \prime \prime}\left(w-3 w^{\prime} w^{\prime \prime}\right)+c w^{\prime \prime \prime}$. Again we can use the translation freedom and absorb the term in $c$ into the term in $a$. Then, inverting $x=1 / w$, the $a$ term disappears, regenerating a term in $c$. Thus, at dominant order, and since the leading term is invariant under inversion, we have for the $N=3$ multilinear mapping the continuous limit $x^{\prime \prime \prime} x^{\prime}-\frac{3}{2} x^{\prime \prime 2}+b x^{\prime \prime \prime}\left(x-3 x^{\prime} x^{\prime \prime}\right)+c x^{\prime \prime \prime}$.

The case of the $N=4$ and $N=5$ mappings can be treated along similar lines. In the case of $N=4$ we find that the dominant terms are $w^{\prime \prime \prime \prime}\left(w^{\prime \prime} w-2 w^{\prime 2}\right)-\frac{4}{3} w^{\prime \prime \prime 2} w+$ $8 x^{\prime \prime \prime} x^{\prime \prime} x^{\prime}-6 w^{\prime \prime 3}$ and $w^{\prime \prime \prime \prime} w^{\prime \prime}-\frac{4}{3} w^{\prime \prime \prime 2}$. Again a translation of $w$ allows to absorb the second term into the first and an inversion of the (translated) $w$ leads to the simple form for the dominant term $x^{\prime \prime \prime \prime} x^{\prime \prime}-\frac{4}{3} x^{\prime \prime \prime 2}$. For $N=5$ there exists only one contribution at dominant

order which reads $x^{(\mathrm{v})}\left(x^{\prime \prime \prime} x^{\prime}-\frac{3}{2} x^{\prime \prime 2}\right)-\frac{5}{4} x^{\prime \prime \prime \prime 2} x^{\prime}+5 x^{\prime \prime \prime \prime} x^{\prime \prime \prime} x^{\prime \prime}-\frac{10}{3} x^{\prime \prime \prime}$. As expected this expression is invariant under inversion.

What is interesting in the continuous limits we examined above is that in each case the leading behaviour is the one predicted by Painlevé and which we encountered in section 2 . This is not in disagreement with the fact that the multilinear mappings are not integrable in general. The conditions that led Painlevé to the form (2.17) are just the first conditions for the Painlevé property to hold and do not suffice for integrability. However we expect the precise continuous limits of the projective systems to fall exactly within the class of integrable equations of Painlevé type (which for orders higher than two have not been fully classified yet).

\section{Conclusion}

In this paper we have examined the alternate forms that can assume projective systems of general orders. We have shown that in both the continuous and the discrete case, it is possible to write a single $N$-th order equation for one of the dependent variables. In the continuous case the differential equation obtained satisfies the necessary integrability conditions established by Painlevé. This is, of course, no surprise, since the projective systems are linearizable and thus integrable by construction. In the discrete case, the mapping obtained belongs to the class of multilinear mappings, which goes beyond the linearizable systems. (The multilinear mappings are not integrable in general, but do contain integrable subclasses). We have explicitly shown that the multilinear projective mapping obtained does satisfy the singularity confinement integrability criterion. Finally, we have 
indicated the procedure for obtaining the continuous limit of these mappings and we have shown that the resulting forms fall within the class obtained by Painlevé.

\section{ACKNOWLEDGEMENTS.}

The financial help of the CEFIPRA, through the contract 1201-1, is gratefully acknowledged. S. Lafortune acknowledges three scholarships. For his Ph.D.: one from NSERC (National Science and Engineering Research Council of Canada) and one from FCAR (Fonds pour la Formation des Chercheurs et l'Aide à la Recherche du Québec). For his stay in Paris: a scholarship from "Programme de Soutien de Cotutelle de Thèse de doctorat du Gouvernement du Québec".

\section{REFERENCES}

[1] P. Painlevé, Acta Math. 25 (1902) 1.

[2] B. Gambier, Acta Math. 33 (1910) 1.

[3] F. Calogero, in What is Integrability?, ed. V. Zakharov, Springer, New York, (1990) 1.

[4] B. Grammaticos, A. Ramani, Meth. Appl. Anal. 4 (1997) 196.

[5] A. Ramani, B. Grammaticos and J. Hietarinta, Phys. Rev. Lett. 67 (1991) 1829.

[6] S. Lafortune, B. Grammaticos, A. Ramani, Inv. Prob. 14 (1998) 287.

[7] B. Grammaticos, A. Ramani, Continuous and discrete linearisable systems: the Riccati saga, Lecture at the Montréal 97 symposium.

[8] B. Grammaticos, A. Ramani and P. Winternitz, Phys. Lett. A 245 (1998) 382.

[9] A. Ramani, B. Grammaticos and G. Karra, Physica A 181 (1992) 115.

[10] R.L. Anderson, J. Harnad and P. Winternitz, Physica D4 (1982) 164-182.

[11] E.L. Ince, Ordinary differential equations, Dover, New York, 1956.

[12] J. Chazy, Acta Math. 34 (1911) 317.

[13] P. Painlevé, C. R. Acad. Sci. (Paris) 130 (1900) 1112.

[14] B. Grammaticos, A. Ramani and V. Papageorgiou, Phys. Rev. Lett. 67 (1991) 1825. 